\title{
EXTENDED REES-SCIAMA EFFECT AND ANISOTROPY OF COSMIC MICROWAVE BACKGROUND RADIATION
}

\author{
X. WU, C. XU AND E. BRÜNING \\ Dept. of Phys., Nanjing Normal Univ., \\ Nanjing 210097, P.R.China
}

The anisotropy of cosmic microwave background radiation (CMBR) was measured by COBE satellite (Smoot et al. 1992). Their remarkable results show a rms-normalized amplitude of $(1.3 \pm 0.4) \times 10^{-5}$ on the scale of $10^{\circ}$ with a thermal spectrum for CMBR quadrupole anisotropy. In near future a new generation of COBE, e.g. COBERAS or Planck (Lubin, 1997) would be lunched, the precision of which is $3-4$ magnitude higher than nowaday COBE's. But the anisotropy can be explained in a different way, since a $10^{-5}$ anisotropy on scale of $10^{\circ}$ might be caused by voids and supercluster (Blumenthal et al., 1992). The additional redshift from voids or supercluster is called Rees-Sciama effects.

In this paper, we used the first order approximate theory for null geodesic line with one perturbed function $u$ as our starting point. By means of null geodesic equation, we deduced a new formalism and established the relation of signals between two points in an expanding and perturbed cosmology. The deducing method is different from usual one. Our formula combine cosmological expanding redshift, gravitational redshift and Rees-Sciama effects in one expression. We obtain the relation between emitting temperature $T_{1}$ and receiving temperature $T_{2}$ as following:

$$
\frac{T_{1}}{T_{2}}=\frac{a\left(t_{2}\right)}{a\left(t_{1}\right)}\left(1-u\left(\mathbf{x}_{1}, t_{1}\right)+u\left(\mathbf{x}_{2}, t_{2}\right)-\frac{2}{a\left(t_{2}\right)} \int_{1}^{2} a(t) \frac{\partial u}{\partial t} d t\right) .
$$

where $u\left(\mathbf{x}_{1}, t_{1}\right), a\left(t_{1}\right)$ and $u\left(\mathbf{x}_{2}, t_{2}\right), a\left(t_{1}\right)$ are the potential and cosmic scale factor at emitting point and receiving point respectively. If we know potential $u$, then we could easily calculate the anisotropy of CMBR which travel in a perturbed R-W metric. In a spherically symmetric overdensity swiss cheese model, we obtain the results similar to Rees-Sciama's original one (Rees, Sciama, 1968). 